\title{
SINGULAR INTEGRALS AND APPROXIMATE IDENTITIES ON SPACES OF HOMOGENEOUS TYPE ${ }^{1}$
}

BY

\author{
HUGO AIMAR
}

\begin{abstract}
In this paper we give conditions for the $L^{2}$-boundedness of singular integrals and the weak type $(1,1)$ of approximate identities on spaces of homogeneous type. Our main tools are Cotlar's lemma and an extension of a theorem of Zó.
\end{abstract}

Introduction. The behavior of singular integrals and approximate identities as operators on the space of integrable functions, i.e. the weak type $(1,1)$, can be investigated by using the Calderon-Zygmund method. This method relies, essentially, on the possibility of solving two problems of different nature:

I. produce an adequate decomposition of $L^{1}$ functions,

II. prove the $L^{p}$ boundedness of the operator for some $p \in(1, \infty]$.

Problem I can be solved in the very general setting of spaces of homogeneous type introduced by $\mathbf{R}$. Coifman and M. de Guzmán in [CG].

In this paper we study problem II and its application to prove the weak type $(1,1)$ of singular integrals and approximate identities operators with kernels defined on spaces of homogeneous type. The approximate identities considered here are natural generalizations to spaces of homogeneous type of those introduced in $[\mathbf{Z}]$. The main results are the $L^{2}$ boundedness of singular integrals and the weak type $(1,1)$ of approximate identities. To prove them we impose an additional geometric condition on the normalized homogeneous structure, that is, the boundedness of the measure of an annulus by the difference of its radii. The precise definition is given in $\S 1$, where we also include several examples of spaces endowed with this property.

The central tool in the proof of $L^{2}$ boundedness of singular integral operators, given in $§ 3$, is Cotlar's lemma. A general class of approximate identities is introduced and studied in $\$ 4$. We use an extension of the theorem of Zó (see [Z]) to the general setting of spaces of homogeneous type. In order to obtain this extension we show in $\$ 2$ a covering lemma and a decomposition lemma for $L^{1}$ functions (i.e. we give a solution for problem I) in the case when the space is not necessarily bounded.

1. Definitions and notation. Let $X$ be a set, let a nonnegative symmetric function $d$ on $X \times X$ be called a quasi-distance if there exists a constant $k$ such that

$$
d(x, y) \leqslant k[d(x, z)+d(z, y)]
$$

for every $x, y, z \in X$, and $d(x, y)=0$ if and only if $x=y$.

Received by the editors September 28, 1984.

1980 Mathematics Subject Classification. Primary 42B20, 42B25.

Key words and phrases. Singular integrals, maximal functions, spaces of homogeneous type.

${ }^{1}$ The content of this paper is part of my Doctoral Dissertation presented at the Universidad de Buenos Aires, June 1983. 
The sets $\{(x, y) \in X \times X: d(x, y)<1 / n\}$ define a base of a metrizable uniform structure on $X$. The balls $B(x, r)=\{y: d(x, y)<r\}$ form a base of neighborhoods of $x$ for the topology induced by the uniform structure.

We shall say that $(X, d, \mu)$ is a space of homogeneous type if $d$ is a quasi-distance on $X, \mu$ is a positive measure defined on a $\sigma$-algebra of subsets of $X$ which contain the balls, and there exists a constant $A$ such that

$$
0<\mu(B(x, 2 r)) \leqslant A \mu(B(x, r))<\infty
$$

holds for every $x \in X$ and $r>0$.

$(X, d, \mu)$ is a bounded space of homogeneous type if there exist $x_{0} \in X$ and $R>0$ such that $X=B\left(x_{0}, R\right)$. In the proof of Lemma (2.1) we shall make use of the following known result: $(X, d, \mu)$ is a bounded space of homogeneous type if and only if $\mu(X)<\infty$. Therefore, if $\mu(X)$ is finite, we shall assume that the radii of balls are bounded.

Following [MS2] we shall say that a space of homogeneous type is normal if there exist finite and positive constants $A_{1}, A_{2}, K_{1}, K_{2}, K_{2} \leqslant 1 \leqslant K_{1}$, such that

$$
\begin{array}{ll}
A_{1} r \leqslant \mu(B(x, r)) & \text { if } r \leqslant K_{1} \mu(X), \\
B(x, r)=X & \text { if } r>K_{1} \mu(X), \\
A_{2} r \geqslant \mu(B(x, r)) & \text { if } r \geqslant K_{2} \mu(\{x\}), \\
B(x, r)=\{x\} & \text { if } r<K_{2} \mu(\{x\})
\end{array}
$$

for every $x \in X$ and $r>0$.

In [MS1], R. Macias and C. Segovia prove that given a space of homogeneous type $(X, d, \mu)$ such that open balls are open sets, a new quasi-distance $\rho$ can be defined on $X$ in such a way that $(X, \rho, \mu)$ is normal and the topologies induced on $X$ by $\rho$ and $d$ coincide. They also prove the following fundamental property for quasi-distances.

If $d$ is a quasi-distance on $X$, then there exist a quasi-distance $d^{\prime}$ equivalent to $d$, a number $\alpha \in(0,1)$ and a finite constant $C$, such that the inequality

$$
\left|d^{\prime}(z, x)-d^{\prime}(z, y)\right| \leqslant C r^{1-\alpha} d^{\prime}(x, y)^{\alpha}
$$

holds whenever $d^{\prime}(z, x)<r$ and $d^{\prime}(z, y)<r$. A space of homogeneous type $\left(X, d^{\prime}, \mu\right)$ satisfying (1.7) shall be called a space of order $\alpha$.

Let $(X, d, \mu)$ be a space of homogeneous type and let $f$ be a locally integrable function on $X$. We write $m_{B}(f)$ for $\mu(B)^{-1} \int_{B} f d \mu$. As in the euclidean case, the weak type $(1,1)$ and the $L^{p}$ boundedness of the Hardy-Littlewood maximal function operator,

$$
M f(x)=\sup \left\{m_{B}(|f|): B \text { is a ball containing } x\right\},
$$

hold (see $[\mathbf{C W}])$.

If $(X, d, \mu)$ is a normal space of homogeneous type, we shall say that $(X, d, \mu)$ satisfies property $\mathrm{P}$ if and only if there exists a finite constant $C$ such that

$$
\mu(B(x, r+s))-\mu(B(x, r)) \leqslant C s
$$

holds for every $x \in X$ and $r, s>0$. 
The following examples show that many of the usual homogeneous structures satisfy this smoothness property.

EXAMPLE 1. If $X=\mathbf{R}^{n}, d(x, y)=|x-y|^{n}$ and $\mu$ is the Lebesgue measure, then $(X, d, \mu)$ is a normal space of order $1 / n$ and satisfies property $\mathrm{P}$.

EXAMPLE 2. Let $X$ be the euclidean space $\mathbf{R}^{n}$ and $\mu$ be the Lebesgue measure. Let $\left\{T_{\lambda}: \lambda>0\right\}$ be a continuous family of transformations on $\mathbf{R}^{n}$ such that $T_{\lambda \sigma}=T_{\lambda} \circ T_{\sigma}$, $T_{1}$ is the identity, and $\left\|T_{\lambda}\right\| \leqslant \lambda$ when $0<\lambda \leqslant 1$. Following [R], we define the distance from $x$ to 0 as the number $\rho=\rho(x)$ such that $\left|T_{\rho^{-1}}(x)\right|=1$. Let $M$ be the matrix such that $T_{\lambda}=e^{M \log \lambda}$ and $\operatorname{tr} M$ be the trace of $M$. Then, the function $d(x, y)=\rho(x-y)^{\operatorname{tr} M}$ is a quasi-distance and $(X, d, \mu)$ is a normal space of order $(\operatorname{tr} M)^{-1}$ satisfying property $\mathrm{P}$. In fact, $\mu(B(X, r))=C r$, where $C$ is a finite constant.

EXAMPLE 3. Let $G$ be a locally compact group and let $\mu$ be the Haar measure on $G$. Assume that $\left\{U_{t}: t>0\right\}$ is a regular Vitali family on $G$ (see $[\mathbf{R}]$ ) such that the sets $U_{t}$ are symmetric neighborhoods of the identity, and $\mu\left(U_{t}\right)$ is continuous and increasing as a function on $\mathbf{R}^{+}$. Then,

$$
d(x, y)=\inf \left\{\mu\left(U_{t}\right): x-y \in U_{t}\right\}
$$

is a quasi-distance on $G$ and $\mu(B(x, r))=r$.

EXAMPLE 4. Let $w$ be a nonnegative locally integrable function defined on $\mathbf{R}$ such that $w(B(x, 2 r)) \leqslant C w(B(x, r))$, where $w(E)=\int_{E} w(x) d x$ and $C$ is a finite constant. This "doubling condition" is satisfied whenever $w$ belongs to a Muckenhoupt's class $A_{p}$. The normalization of the space $(\mathbf{R},|\cdot|, w d x)$ gives the distance

$$
d(x, y)=\left|\int_{x}^{y} w(z) d z\right| .
$$

It is easy to prove that $w\left(B_{d}(x, r)\right)=2 r$. In particular, property P holds.

EXAMPLE 5. In order to obtain the results on approximate identities included in $\S 4$, the property of symmetry for the quasi-distance can be replaced by the existence of two constants $C_{1}$ and $C_{2}$ such that

$$
C_{1} d(x, y) \leqslant d(y, x) \leqslant C_{2} d(x, y)
$$

holds for every $x, y \in X$. An example of a quasi-distance satisfying this weak symmetry is given by

$$
d(x, y)=w(B(x,|x-y|)),
$$

where $w$ is a weight function on $\mathbf{R}^{n}$ satisfying a doubling condition and $B(x,|x-y|)$ is a euclidean ball. If $B_{d}(x, r)=\{y: d(x, y)<r\}$, then $w\left(B_{d}(x, r)\right)=r$. We can prove that $\left(\mathbf{R}^{n}, d, w d x\right)$ is also of order $\alpha$ in the sense that (1.7) holds. This is an easy consequence of the $\beta$ property proved in [GGW], namely, there exists $\beta \in[0,1)$ and a finite constant $C$ such that

$$
\begin{aligned}
& w\left(B\left(x,\left|x-x_{0}\right|+s\right)\right)-w\left(B\left(x,\left|x-x_{0}\right|\right)\right) \\
& \leqslant C w\left(B\left(x_{0}, s\right)\right)^{1-\beta} w\left(B\left(x,\left|x-x_{0}\right|\right)\right)^{\beta}
\end{aligned}
$$

holds whenever $\left|x-x_{0}\right| \geqslant s$. 
2. Basic lemmas. A covering lemma for bounded sets on spaces of homogeneous type can be found in [CW]. In order to prove a Calderón-Zygmund type lemma for nonnecessarily bounded spaces of homogeneous type, we need the following

(2.1) Covering Lemma. Let $(X, d, \mu)$ be a space of homogeneous type. Let $\mathscr{B}=\left\{B_{\alpha}: \alpha \in \Gamma\right\}$ be a family of balls such that the set $E=\bigcup_{\alpha \in \Gamma} B_{\alpha}$ is measurable and $\mu(E)<\infty$. Then there exists a disjoint sequence $\left\{B_{i}\right\}=\left\{B\left(x_{i}, r_{i}\right)\right\} \subset \mathscr{B}$ such that $E \subset \cup B\left(x_{i}, C r_{i}\right)$, with a constant $C$ depending only on $k$. Moreover, every $B \in \mathscr{B}$ is contained in some $B\left(x_{i}, C r_{i}\right)$.

Proof. Observe that if $\Lambda \subset \Gamma$ and $B_{\lambda}=B\left(x_{\lambda}, r_{\lambda}\right)$ is a fixed ball with $\lambda \in \Lambda$, then the family

$$
\mathscr{F}=\left\{B_{\alpha}: \alpha \in \Lambda \text { and } B\left(x_{\alpha}, 2 k r_{\alpha}\right) \cap B_{\lambda} \neq \varnothing\right\}
$$

is nonempty and the set $\mathscr{R}=\left\{r_{\alpha}: B_{\alpha} \in \mathscr{F}\right\}$ is bounded. In fact, if $X$ is bounded there is nothing to prove. Assume that $X$ is unbounded and $\sup \mathscr{R}=\infty$. Let $\left\{r_{j}\right\} \subset \mathscr{R}$ be an increasing sequence such that $r_{j}>r_{\lambda}$ for every $j$ and $r_{j}$ tends to $\infty$ when $j$ tends to $\infty$. From $B_{\lambda} \cap B\left(x_{j}, 2 k r_{j}\right) \neq \varnothing$ we deduce easily that $B\left(x_{\lambda}, r_{j}\right) \subset$ $B\left(x_{j}, 4 k^{3} r_{j}\right)$. Thus, applying (1.2), it follows that

$$
\mu\left(B\left(x_{\lambda}, r_{j}\right)\right) \leqslant C \mu\left(B\left(x_{j}, r_{j}\right)\right) \leqslant C \mu(E)<\infty,
$$

which is a contradiction because $\mu(X)=\infty$ and the left-hand side increases to $\mu(X)$.

The sequence $\left\{B_{i}\right\}$ can be constructed inductively in the following way: Let $\mathscr{B}_{1}=B$ and $B_{0,1}=B\left(x_{0,1}, r_{0,1}\right) \in \mathscr{B}_{1}$ such that

$$
2 \mu\left(B_{0,1}\right)>\sup \left\{\mu(B): B \in \mathscr{B}_{1}\right\} .
$$

Let

$$
\tilde{\mathscr{B}}_{1}=\left\{B_{\alpha}=B\left(x_{\alpha}, r_{\alpha}\right) \in \mathscr{B}_{1}: B\left(x_{\alpha}, 2 k r_{\alpha}\right) \cap B_{0,1} \neq \varnothing\right\} .
$$

Therefore, the set $\mathscr{R}_{1}=\left\{r_{\alpha}: B_{\alpha} \in \tilde{\mathscr{B}}_{1}\right\}$ is bounded and, consequently, we can choose $B_{1}=B\left(x_{1}, r_{1}\right) \in \mathscr{B}_{1}$ such that $2 r_{1}>\sup \mathscr{R}_{1}$. Let us prove that if $B=$ $B(x, r) \in \mathscr{B}_{1}$ and $B \cap B_{1} \neq \varnothing$ then $B \subset B\left(x_{1}, C r_{1}\right)$ for some constant $C$ depending only on $k$. Assume that $r \leqslant k(1+2 k) r_{1}$. Let $z \in B$ and $u \in B \cap B_{1}$. Applying (1.1) we obtain

$$
d\left(z, x_{1}\right) \leqslant k\left[d(z, x)+k\left(d(x, u)+d\left(u, x_{1}\right)\right)\right]<k\left[r+k\left(r+r_{1}\right)\right] \leqslant C r_{1},
$$

which proves our assertion when $r \leqslant k(1+2 k) r_{1}$. But this is always the case, otherwise, if $y \in B\left(x_{1}, 2 k r_{1}\right) \cap B_{0,1}$ and $u \in B \cap B_{1}$, the inequality

$$
d(x, y) \leqslant k\left[d(x, u)+k\left(d\left(u, x_{1}\right)+d\left(x_{1}, y\right)\right)\right]<k\left[r+k\left(r_{1}+2 k r_{1}\right)\right]<2 k r
$$

proves that $B \in \tilde{\mathscr{B}}_{1}$. From this we get

$$
2 r_{1}>\sup \mathscr{R}_{1} \geqslant r>k(1+2 k) r_{1} \geqslant 3 r_{1},
$$

which is a contradiction. 
Assume $B_{j}=B\left(x_{j}, r_{j}\right)$ and $B_{0, j}=B\left(x_{0, j}, r_{0, j}\right), j=1,2, \ldots i$, are given satisfying

$$
\begin{gathered}
B_{0, j} \in \mathscr{B}_{j}=\left\{B \in \mathscr{B}: B \cap\left[B_{1} \cup \cdots \cup B_{j-1}\right]=\varnothing\right\}, \\
2 \mu\left(B_{0, j}\right)>\sup \left\{\mu(B): B \in \mathscr{B}_{j}\right\}, \\
B_{j} \in \tilde{\mathscr{B}}_{j}=\left\{B_{\alpha}=B\left(x_{\alpha}, r_{\alpha}\right) \in \mathscr{B}_{j}: B\left(x_{\alpha}, 2 k r_{\alpha}\right) \cap B_{0, j} \neq \varnothing\right\}, \\
2 r_{j}>\sup \mathscr{R}_{j}, \quad \text { where } \mathscr{R}_{j}=\left\{r_{\alpha}: B\left(x_{\alpha}, r_{\alpha}\right) \in \tilde{B}_{j}\right\},
\end{gathered}
$$

if $B \in \mathscr{B}_{j}$ and $B \cap B_{j} \neq \varnothing$, then $B \subset B\left(x_{j}, C r_{j}\right)$, where $C$ is a constant depending only on $k$.

Suppose there exists $\alpha \in \Gamma$ such that there is no $j=1, \ldots, i$ for which $B_{\alpha} \subset$ $B\left(x_{j}, C r_{j}\right)$. Clearly, $\mathscr{B}_{i+1} \neq \varnothing$. Let us pick $B_{0, i+1} \in \mathscr{B}_{i+1}$ such that

$$
2 \mu\left(B_{0,1}\right)>\sup \left\{\mu(B): B \in \mathscr{B}_{i+1}\right\} \text {. }
$$

On account of the boundedness of $\mathscr{R}_{i+1}$ we can choose $B_{i+1}=B\left(x_{i+1}, r_{i+1}\right) \in \tilde{\mathscr{B}}_{i+1}$ with $2 r_{i+1}>\sup \mathscr{R}_{i+1}$. The proof of (2.6) for $j=i+1$ is like the one for the case $j=1$.

If in some step $i$ of the induction process every $B_{\alpha}$ is contained in some $B\left(x_{j}, C r_{j}\right)$, $j=1, \ldots, i$, then the finite sequence $\left\{B_{1}, \ldots, B_{i}\right\}$ satisfies the required properties. If this is not the case, we get a disjoint infinite sequence $\left\{B_{i}\right\}$. Let $B \in \mathscr{B}$. If we show that $B \cap \bigcup_{i=1}^{\infty} B_{i} \neq \varnothing$, then the result follows from (2.6). Assume that $B \cap \bigcup_{i=1}^{\infty} B_{i}$ $=\varnothing ;$ then $B \in \mathscr{B}_{i}$ for every $i \geqslant 1$. From (2.5) we get $2 r_{i}>r_{0, i}$, consequently $B_{0, i} \subset B\left(x_{i}, 6 k^{3} r_{i}\right)$. Finally, taking into account (2.3), we have

$$
0<\mu(B)<2 \mu\left(B_{0, i}\right) \leqslant C \mu\left(B_{i}\right) \text {. }
$$

Thus

$$
\mu(E) \geqslant \mu\left(\bigcup_{i=1}^{\infty} B_{i}\right)=\sum_{i=1}^{\infty} \mu\left(B_{i}\right)=\infty,
$$

which is a contradiction. This finishes the proof of the lemma.

(2.7) LeMma. Let $(X, d, \mu)$ be a space of homogeneous type such that the open balls are open sets. Let $f$ be a nonnegative integrable function defined on $X$. Then for every $\lambda \geqslant m_{X}(f)\left(m_{X}(f)=0\right.$ if $\left.\mu(X)=\infty\right)$, there exists a sequence of disjoint balls, $\left\{B_{i}\right\}=\left\{B\left(x_{i}, r_{i}\right)\right\}$ such that, if $\tilde{B}_{i}=B\left(x, C r_{i}\right), C$ the constant in Lemma (2.1),

$$
m_{\tilde{B}_{i}}(f) \leqslant \lambda<m_{B_{i}}(f),
$$

$$
m_{B}(f) \leqslant \lambda \quad \text { for every ball } B \text { centered at } x \in X-\cup_{i} \tilde{B}_{i} .
$$

Proof. Let $\Omega=\left\{x \in X: m_{B(x, r)}(f)>\lambda\right.$ for some $\left.r>0\right\}$. If $\Omega=\varnothing$, then (2.9) holds for every $x \in X$ and the lemma follows. Let $x \in \Omega$. The integrability of $f$ implies that the set $\left\{r>0: m_{B(x, r)}(f)>\lambda\right\}$ is bounded. Consequently we can choose $r(x)>0$ such that

$$
m_{B(x, r(x))}(f)>\lambda \geqslant m_{B(x, C r(x))}(f) .
$$

The set $E=\bigcup_{x \in \Omega} B(x, r(x))$ is open and, in particular, measurable. From the weak type $(1,1)$ for the Hardy-Littlewood maximal operator we deduce that

$$
\mu(E)=\mu(\{y \in X: M f(y)>\lambda\}) \leqslant \frac{C}{\lambda} \int_{X} f d \mu<\infty .
$$


We now apply Lemma (2.1) in order to get a sequence $\left\{B_{i}\right\}$, which clearly satisfies (2.8) and (2.9).

This result allows us to obtain a Calderón-Zygmund type decomposition for $L^{1}$ functions. In fact, preserving the notation of Lemma (2.7) we have

(2.10) Calderon-Zygmund Type Lemma. Let $(X, d, \mu)$ be a space of homogeneous type such that the open balls are open sets and the continuous functions are dense in $L^{1}$. Let $f$ be a nonnegative integrable function and $\lambda \geqslant m_{X}(f)$. Then there exist functions $h_{i}$ such that the sets $S_{i}=\left\{x: h_{i}(x) \neq 0\right\}$ are pairwise disjoint and

$$
\begin{gathered}
g=f-\sum_{i} h_{i} \in L^{1} \cap L^{\infty} \text { and }\|g\|_{\infty} \leqslant D \lambda, \\
\int h_{i} d \mu=0, \\
S_{i} \subset \tilde{B}_{i}, \\
\sum_{i} \mu\left(\tilde{B}_{i}\right) \leqslant D \lambda^{-1}\|f\|_{1},
\end{gathered}
$$

where the constant $D$ depends only on $k$ and $A$.

Proof. Observe that the sequence $\left\{V_{i}\right\}$, defined by

$$
V_{1}=\tilde{B}_{1}-\bigcup_{n=2}^{\infty} B_{n}, \quad V_{i}=\tilde{B}_{i}-\left[\bigcup_{n=1}^{i-1} V_{n} \cup \bigcup_{n=i+1}^{\infty} B_{n}\right],
$$

satisfies the properties

$$
B_{i} \subset V_{i} \subset \tilde{B}_{i} \text { and } \bigcup_{i} V_{i}=\bigcup_{i} \tilde{B}_{i}
$$

Let $h_{i}(x)=\left[f(x)-m_{V_{i}}(f)\right] \chi_{V_{i}}(x)$, where $\chi_{E}$ denotes the characteristic function of the set $E$. Clearly $h_{i}$ satisfies (2.12) and (2.13).

From (2.8) we deduce (2.14) in the following way:

$$
\sum_{i} \mu\left(\tilde{B}_{i}\right) \leqslant D \sum_{i} \mu\left(B_{i}\right)<D \lambda^{-1} \sum_{i} \int_{B_{i}} f d \mu \leqslant D \lambda^{-1}\|f\|_{1} .
$$

In order to prove (2.11) let us first assume $x \notin \bigcup_{i} V_{i}$. Since the continuous functions are dense in $L^{1}(X)$, the Lebesgue differentiation theorem applies. Therefore, using (2.9) we get $g(x)=f(x) \leqslant \lambda$. Taking now $x \in V_{i}$, from (2.8) we obtain

$$
g(x)=m_{V_{i}}(f) \leqslant \frac{\mu\left(\tilde{B}_{i}\right)}{\mu\left(V_{i}\right)} m_{\tilde{B}_{i}}(f) \leqslant \frac{\mu\left(\tilde{B}_{i}\right)}{\mu\left(B_{i}\right)} m_{\tilde{B}_{i}}(f) \leqslant D \lambda .
$$

In a metric space, if $z$ belongs to a ball $B(y, r)$, then

$$
B(z, r-d(z, y)) \subset B(y, r) \subset B(z, r+d(z, y)) .
$$

A simple and useful analogous property holds on spaces of homogeneous type of order $\alpha$.

(2.15) Lemma. Let $(X, d, \mu)$ be a space of homogeneous type of order $\alpha$. Let $k$ and $C$ be the constants in (1.1) and (1.7) respectively. Then, given $z, y \in X$ and $r>0$ such that

$$
d(z, y)<\left[C \cdot(2 k)^{1-\alpha}\right]^{-1 / \alpha} r
$$


we have

$$
\varnothing \neq B\left(z, r-\delta r^{1-\alpha} d(z, y)^{\alpha}\right) \subset B(y, r) \subset B\left(z, r+\delta r^{1-\alpha} d(z, y)^{\alpha}\right)
$$

for every $\delta$ satisfying $C(2 k)^{1-\alpha} \leqslant \delta<[r / d(z, y)]^{\alpha}$.

Proof. Since

$$
r-\delta r^{1-\alpha} d(z, y)^{\alpha}>r-[r / d(z, y)]^{\alpha} r^{1-\alpha} d(z, y)^{\alpha}=0,
$$

we have $B\left(z, r-\delta r^{1-\alpha} d(z, y)^{\alpha}\right) \neq \varnothing$. From (2.16) we see that $d(z, y)<r$. Let $u \in B\left(z, r-\delta r^{1-\alpha} d(z, y)^{\alpha}\right)$. Then $d(u, z)<r$ and $d(u, y) \leqslant 2 k r$, and we can apply the property of order $\alpha$ in order to obtain

$$
\begin{aligned}
d(u, y) & \leqslant d(u, z)+C(2 k)^{1-\alpha} r^{1-\alpha} d(y, z)^{\alpha} \\
& <r+\left[C(2 k)^{1-\alpha}-\delta\right] r^{1-\alpha} d(y, z)^{\alpha} \leqslant r,
\end{aligned}
$$

which proves the first inclusion; the second can be proved similarly.

We shall frequently use the following known properties on the integrability of powers of the quasi-distance on normal spaces of homogeneous type.

Lemma. Let $(X, d, \mu)$ be a normal space of homogeneous type. Let $r>0$ and $x \in X$. Then

$$
\begin{gathered}
\int_{d(x, y)<r} d(x, y)^{-1} d \mu(y)=\infty, \\
\int_{d(x, y) \geqslant r} d(x, y)^{-1} d \mu(y)=\infty \quad \text { if } \mu(X)=\infty .
\end{gathered}
$$

If $\mu(\{x\})=0$ and $\alpha>0$, then there exists a constant $C$ such that

$$
\begin{gathered}
\int_{d(x, y) \geqslant r} d(x, y)^{-1-\alpha} d \mu(y) \leqslant C r^{-\alpha}, \\
\int_{d(x, y)<r} d(x, y)^{\alpha-1} d \mu(y) \leqslant C r^{\alpha} .
\end{gathered}
$$

3. Singular integrals. In this section $(X, d, \mu)$ is a normal space of homogeneous type and $k$ is the constant in (1.1). We shall consider a singular integral operator with measurable kernel $K: X \times X \rightarrow \mathbf{R}$ satisfying the following properties:

(3.1) There exists a constant $C_{1}$ such that $|K(x, y)| \leqslant C_{1} d(x, y)^{-1}$ holds for every $x \neq y$.

(3.2) There exist $\alpha \in(0,1)$ and $C_{2}>0$ such that

(a) $|K(y, x)-K(z, x)| \leqslant C_{2} d(y, z)^{\alpha} d(x, y)^{-1-\alpha}$,

(b) $|K(x, y)-K(x, z)| \leqslant C_{2} d(y, z)^{\alpha} d(x, y)^{-1-\alpha}$ hold if $d(x, y)>2 d(y, z)$.

(3.3) For every $R$ and $r$ we have

(a) $\int_{r \leqslant d(x, y)<R} K(x, y) d \mu(y)=0$, for every $x \in X$,

(b) $\int_{r \leqslant d(x, y)<R} K(x, y) d \mu(x)=0$, for every $y \in X$.

Given $R>r>0$, define

$$
K_{R, r} f(x)=\int_{r \leqslant d(x, y)<R} K(x, y) f(y) d \mu(y),
$$


where $f$ is a locally integrable function. If $i \in \mathbf{Z}$ we write $\chi_{i}$ for the characteristic function of the set

$$
\left\{(x, y) \in X \times X:(2 k)^{i} \leqslant d(x, y)<(2 k)^{i+1}\right\}
$$

and $K_{i}$ for $K \chi_{i}$.

(3.4) Lemma. Let $(\chi, d, \mu)$ be a space of order $\alpha$ satisfying Property P. If $K$ is a singular kernel for which (3.1) and (3.2)(a) hold, then there exists a finite constant $C_{3}$ such that

$$
\int_{X}\left|K_{i}(y, x)-K_{i}(z, x)\right| d \mu(x) \leqslant C_{3}(2 k)^{-\alpha i} d(y, z)^{\alpha}
$$

holds for every $i \in \mathbf{Z}$ and $y, z \in X$.

Proof. Let $C$ be the constant in (1.7), $x, y \in X$ and $i \in \mathbf{Z}$. Assume

$$
(2 k)^{\alpha i} d(y, z)^{-\alpha} \leqslant 4 C(2 k)^{1-\alpha} \text {. }
$$

In this case the inequality (3.5) follows readily from (3.1). Indeed

$$
\begin{aligned}
\int_{X}\left|K_{i}(y, x)-K_{i}(z, x)\right| d \mu(x) \leqslant & C_{1} \int_{(2 k)^{i} \leqslant d(x, y)<(2 k)^{i+1}} d(x, y)^{-1} d \mu(x) \\
& +C_{1} \int_{(2 k)^{\prime} \leqslant d(x, z)<(2 k)^{i+1}} d(x, z)^{-1} d \mu(x) .
\end{aligned}
$$

Then on account of (1.5), we have that the left-hand side in (3.5) is bounded above. From our assumption the right-hand side of (3.5) is bounded below by a positive constant. Thus, (3.5) holds. Suppose $(2 k)^{\alpha i} d(y, z)^{-\alpha}>4 C(2 k)^{1-\alpha}$. Let

$$
\mathrm{I}=\int_{(2 k)^{i} \leqslant d(x, y)<(2 k)^{i+1}}|K(y, x)-K(z, x)| d \mu(x)
$$

and

$$
\mathrm{II}=\int_{X}|K(z, x)|\left|\chi_{i}(y, x)-\chi_{i}(z, x)\right| d \mu(x) .
$$

Therefore

$$
\int_{X}\left|K_{i}(y, x)-K_{i}(z, x)\right| d \mu(x) \leqslant \mathrm{I}+\mathrm{II} .
$$

In order to estimate I, from

$$
(2 k)^{i}>d(y, z)\left[4 C(2 k)^{1-\alpha}\right]^{1 / \alpha}>2 d(y, z),
$$

using (3.2)(a), we get

$$
\begin{aligned}
\mathrm{I} & \leqslant C_{2} d(y, z)^{\alpha} \int_{(2 k)^{\prime} \leqslant d(x, y)<(2 k)^{i+1}} d(x, y)^{-1-\alpha} d \mu(x) \\
& \leqslant C(2 k)^{-\alpha i} d(y, z)^{\alpha} .
\end{aligned}
$$

On the other hand, clearly

$$
\mathrm{II}=\int_{د,(y, z)}|K(z, x)| d \mu(x),
$$


where

$$
\Delta_{i}(y, z)=\left[B\left(y,(2 k)^{i+1}\right)-B\left(y,(2 k)^{i}\right)\right] \Delta\left[B\left(z,(2 k)^{i+1}\right)-B\left(z,(2 k)^{i}\right)\right]
$$

and $\Delta$ denotes the symmetric difference. Applying Lemma (2.15) we shall see that $\Delta_{i}(y, z)$ is included in the union of two annuli centered at $z$. Let $r_{1}=(2 k)^{i}$ and $r_{2}=(2 k)^{i+1}$. Then

$$
\begin{aligned}
d(z, y) & \leqslant\left[4 C(2 k)^{1-\alpha}\right]^{-1 / \alpha}(2 k)^{i}<\left[C(2 k)^{1-\alpha}\right]^{-1 / \alpha} r_{1} \\
& <\left[C(2 k)^{1-\alpha}\right]^{-1 / \alpha} r_{2},
\end{aligned}
$$

which is (2.16) for $r_{1}$ and $r_{2}$. Letting $a_{i}=(2 k)^{i}-\delta(2 k)^{i(1-\alpha)} d(z, y)^{\alpha}$ and $b_{i}=$ $(2 k)^{i}+\delta(2 k)^{i(1-\alpha)} d(z, y)^{\alpha}$, with $\delta=2 C(2 k)^{1-\alpha}$, we have

$$
B\left(z, a_{i}\right) \subset B\left(y,(2 k)^{i}\right) \subset B\left(z, b_{i}\right)
$$

and

$$
B\left(z, a_{i+1}\right) \subset B\left(y,(2 k)^{i+1}\right) \subset B\left(z, b_{i+1}\right) .
$$

Consequently,

$$
\Delta_{i}(y, z) \subset\left[B\left(z, b_{i+1}\right)-B\left(z, a_{i+1}\right)\right] \cup\left[B\left(z, b_{i}\right)-B\left(z, a_{i}\right)\right] .
$$

So applying (3.1) we obtain

$$
\begin{aligned}
\mathrm{II} \leqslant & C_{1} a_{i+1}^{-1}\left[\mu\left(B\left(z, b_{i+1}\right)\right)-\mu\left(B\left(z, a_{i+1}\right)\right)\right] \\
& +C_{1} a_{i}^{-1}\left[\mu\left(B\left(z, b_{i}\right)\right)-\mu\left(B\left(z, a_{i}\right)\right)\right] .
\end{aligned}
$$

On account of property $\mathrm{P}$, the right-hand side in the above inequality is bounded by

$$
\begin{aligned}
C & {\left[a_{i+1}^{-1}\left(b_{i+1}-a_{i+1}\right)+a_{i}^{-1}\left(b_{i}-a_{i}\right)\right] } \\
& =C\left[\frac{2 \delta(2 k)^{(i+1)(1-\alpha)} d(z, y)^{\alpha}}{(2 k)^{i+1}-\delta(2 k)^{(i+1)(1-\alpha)} d(z, y)^{\alpha}}+\frac{2 \delta(2 k)^{i(1-\alpha)} d(z, y)^{\alpha}}{(2 k)^{i}-\delta(2 k)^{i(1-\alpha)} d(z, y)^{\alpha}}\right] \\
& \leqslant C(2 k)^{-\alpha i} d(z, y)^{\alpha},
\end{aligned}
$$

finishing the proof of the lemma.

REMARK. If the kernel $K$ satisfies (3.2)(b) instead of (3.2)(a), we get

$$
\int_{X}\left|K_{i}(x, y)-K_{i}(x, z)\right| d \mu(x) \leqslant C_{3}(2 k)^{-\alpha i} d(y, z)^{\alpha} .
$$

CotlaR's Lemma. Let $H$ be a Hilbert space and $T_{1}, T_{2}, \ldots, T_{N}$ a finite sequence of linear and continuous operators on $H$. Let $c: \mathbf{Z} \rightarrow[0, \infty)$ such that $\sum_{l=-\infty}^{\infty} c(l)^{1 / 2}=A$ $<\infty$ and let $T_{i}^{*}$ be the adjoint of $T_{i}$. If $\left\|T_{i}{ }^{*} T_{j}\right\| \leqslant c(i-j)$ and $\left\|T_{i} T_{j}{ }^{*}\right\| \leqslant c(i-j)$, then $\left\|\sum_{i=1}^{N} T_{i}\right\| \leqslant A$.

This version of Cotlar's Lemma and its proof can be found in [G].

(3.6) TheOREM. Let $(X, d, \mu)$ be as in lemma (3.4) and let $K$ be a singular kernel satisfying (3.1), (3.2) and (3.3). Then there exists $C$, independent on $R, r$ and $f$, such that $\left\|K_{R, r} f\right\|_{2} \leqslant C\|f\|_{2}$. 
Proof. If $f \in L^{2}(X, \mu)$ and $x \in X$, then $K_{j}(x, y) f(y)$ is an absolutely integrable function of $y$. Consequently, the function $T_{j} f(x)=\int K_{j}(x, y) f(y) d \mu(y)$ is well defined. Moreover, $T_{j}$ is linear and continuous as an operator on $L^{2}$. In fact, applying Schwartz's inequality and (3.1) we get

$$
\begin{aligned}
\left|T_{j} f(x)\right|^{2} & \leqslant\left\{\int\left|K_{j}(x, y)\right||f(y)|^{2} d \mu(y)\right\} \cdot\left\{\int\left|K_{j}(x, y)\right| d \mu(y)\right\} \\
& \leqslant C \int\left|K_{j}(x, y)\right||f(y)|^{2} d \mu(y)
\end{aligned}
$$

so that

$$
\left\|T_{j} f\right\|_{2}^{2} \leqslant C \int|f(y)|^{2}\left\{\int\left|K_{j}(x, y)\right| d \mu(x)\right\} d \mu(y) \leqslant C\|f\|_{2}^{2} .
$$

The adjoint $T_{j}^{*}$ of $T_{j}$ is the integral operator with kernel $\tilde{K}_{j}(x, y)=K_{j}(y, x)$, i.e.

$$
T_{j}^{*} g(x)=\int K_{j}(y, x) g(y) d \mu(y) .
$$

In order to apply Cotlar's Lemma, we shall estimate the norm of $T_{i}{ }^{*} T_{j}$. Since $(X, \mu)$ is $\sigma$-finite, from Fubini's theorem we see that $T_{i}^{*} T_{j}$ is the integral operator with kernel $\int K_{i}(y, x) K_{j}(y, z) d \mu(y)$, namely

$$
T_{i}^{*} T_{j} f(x)=\int\left\{\int K_{i}(y, x) K_{j}(y, z) d \mu(y)\right\} f(z) d \mu(z),
$$

where $f \in L^{2}$. Applying Schwartz's inequality, the function $\left|T_{i}{ }^{*} T_{j} f(x)\right|^{2}$ is majorized by

$$
\begin{array}{r}
\left\{\int\left|\int K_{i}(y, x) K_{j}(y, z) d \mu(y)\right||f(z)|^{2} d \mu(z)\right\} \\
\quad \times\left\{\int\left|\int K_{i}(y, x) K_{j}(y, z) d \mu(y)\right| d \mu(z)\right\} .
\end{array}
$$

Assume that $i \geqslant j$. On account of (3.1), the second factor in (3.7) is bounded by a constant $C$ depending only on $k, A$ and $C_{1}$. Applying (3.3), Lemma (3.4) and (3.1), we have

$$
\begin{aligned}
\left\|T_{i}^{*} T_{j} f\right\|_{2}^{2} & \leqslant C \int\left\{\int\left|\int K_{i}(y, x) K_{j}(y, z) d \mu(y)\right||f(z)|^{2} d \mu(z)\right\} d \mu(x) \\
& =C \int\left\{\int\left|\int\left[K_{i}(y, x)-K_{i}(z, x)\right] K_{j}(y, z) d \mu(y)\right||f(z)|^{2} d \mu(z)\right\} d \mu(x) \\
& \leqslant C \int|f(z)|^{2} \int_{1 \leqslant d(y, z) /(2 k)^{\prime} \leqslant 2 k} d(y, z)^{\alpha-1}(2 k)^{-\alpha i} d \mu(y) d \mu(z) \\
& \leqslant C(2 k)^{-\alpha i}(2 k)^{(\alpha-1) j}(2 k)^{j+1}\|f\|_{2}^{2} \\
& =C(2 k)^{-\alpha|i-j|}\|f\|_{2}^{2} .
\end{aligned}
$$

Therefore, we get

$$
\left\|T_{i}^{*} T_{j}\right\| \leqslant C(2 k)^{-\alpha|i-j| / 2}
$$


for $i \geqslant j$. If $i<j$, we apply (3.3), (3.5) and (3.1) in order to estimate the second factor of (3.7):

$$
\begin{aligned}
& \int\left|\int K_{i}(y, x) K_{j}(y, z) d \mu(y)\right| d \mu(z) \\
& \quad=\int\left|\int K_{i}(y, x)\left[K_{j}(y, z)-K_{j}(x, z)\right] d \mu(y)\right| d \mu(z) \\
& \quad \leqslant C \int_{1 \leqslant d(x, y) /(2 k)^{i}<2 k} d(x, y)^{\alpha-1}(2 k)^{-\alpha j} d \mu(y) \\
& \quad \leqslant C(2 k)^{-\alpha|i-j|},
\end{aligned}
$$

so that

$$
\begin{aligned}
\left\|T_{i}^{*} T_{j} f\right\|_{2}^{2} & \leqslant C(2 k)^{-\alpha|i-j|} \int|f(z)|^{2} \int\left|K_{j}(y, z)\right| \int\left|K_{i}(y, x)\right| d \mu(x) d \mu(y) d \mu(z) \\
& \leqslant C(2 k)^{-\alpha|i-j|}\|f\|_{2}^{2}
\end{aligned}
$$

which proves that (3.8) also holds for $i<j$. By the symmetry on the properties of $K$, the same estimate holds for $\left\|T_{i} T_{j}^{*}\right\|$. Letting $c(l)=C(2 k)^{-\alpha \mid l / / 2}$ and applying Cotlar's Lemma we obtain

$$
\left\|\sum_{l=i}^{j} T_{l}\right\| \leqslant \sum_{l \in \mathbf{Z}} c(l)^{1 / 2}<\infty
$$

for every $i<j$. If $0<r<R<\infty$, let $i, j \in \mathbf{Z}$ such that $(2 k)^{i} \leqslant r<(2 k)^{i+1}$ and $(2 k)^{j} \leqslant R<(2 k)^{j+1}$. Then, from (3.1), we get

$$
\begin{aligned}
\left|K_{R, r} f(x)\right| \leqslant & \int_{\left\{(2 k)^{j} \leqslant d(x, y)<R\right\} \cup\left\{r \leqslant d(x, y)<(2 k)^{i+1}\right\}}|K(x, y)||f(y)| d \mu(y) \\
& +\sum_{l=i+1}^{j-1} T_{l} f(x) \\
\leqslant & C M f(x)+\sum_{l=i+1}^{j-1} T_{l} f(x) .
\end{aligned}
$$

Therefore $\left\|K_{R, r} f\right\|_{2} \leqslant C\|f\|_{2}$.

REMARK 1. The fact that the order of the space and the order of the smoothness condition (3.2) coincide is clearly nonrestrictive, since we could use the smaller one.

REMARK 2. In general for $1<p<\infty$, if we know that, for instance, a Lipschitz class is dense in $L^{p}(X)$, we can obtain the usual results on $L^{p}$ and pointwise convergence for $K_{R, r}(f)$ by a suitable modification of the standard argument. This is the case if, for example, $(X, \mu)$ is a regular measure space, since then there exists $\alpha \in(0,1)$ such that the class Lipschitz $\alpha$ is dense in $L^{p}(X)$.

4. Approximate identities. We first give a generalization to spaces of homogeneous type of some results due to F. Zó (see [Z]) on sufficient conditions for the weak type $(1,1)$ of the maximal operator associated to a family of integrable kernels. 
(4.1) TheOrem. Let $(X, d, \mu)$ be a space of homogeneous type such that continuous functions are dense in $L^{1}$. Let $T$ be a sublinear and countably subadditive operator, from $L^{1}+L^{\infty}$ into the space of measurable functions on $X$. Assume there exist $M$ and $C_{0}$ satisfying

$$
\begin{gathered}
\|T g\|_{\infty} \leqslant C_{0}\|g\|_{\infty}, \\
\int_{X-B\left(x_{0}, M r\right)}|T h(x)| d \mu(x) \leqslant C_{0} \int_{X}|h(x)| d \mu(x)
\end{gathered}
$$

for $h \in L^{1},\{h \neq 0\} \subset B\left(x_{0}, r\right)$ and $\int h d \mu=0$. Then $T$ is of weak type $(1,1)$ and of strong type $(p, p)(1<p \leqslant \infty)$.

Proof. Let $\rho$ be a quasi-distance on $X$ such that the $\rho$-balls are open sets and $C_{1} \rho(x, y) \leqslant d(x, y) \leqslant C_{2} \rho(x, y)$ for some finite constants $C_{1}, C_{2}$ and every $x$, $y \in X$. If $f$ is a nonnegative integrable function with $m_{X}(f) \leqslant 1$ we can apply Lemma (2.10) with $\lambda=1$ and $f$ considered as a function on $(X, \rho, \mu)$. In this way we can write $f=g+\sum_{n} h_{n}$, where $g$ and $h_{n}$ satisfy (2.11)-(2.14). On account of (4.2) and the sublinearity of $T$, we only need to show

$$
\mu\left(\left\{x \in X:\left|T\left(\sum h_{n}\right)(x)\right|>C_{0} D\right\}\right) \leqslant c\|f\|_{1} .
$$

Let $C$ be the constant in Lemma (2.1). Clearly,

$$
\begin{aligned}
\mu(\{x \in & \left.\left.X:\left|T\left(\sum h_{n}\right)(x)\right|>C_{0} D\right\}\right) \\
\leqslant & \mu\left(\left\{x \notin \bigcup_{n} B_{d}\left(x_{n}, M C C_{2} r_{n}\right):\left|T\left(\sum h_{n}\right)(x)\right|>C_{0} D\right\}\right) \\
& +\mu\left[\bigcup_{n} B_{d}\left(x_{n}, M C C_{2} r_{n}\right)\right] .
\end{aligned}
$$

Since $B_{d}\left(x_{n}, M C C_{2} r_{n}\right) \subset B_{\rho}\left(x_{n}, M C C_{2} C_{1}^{-1} r_{n}\right)$, from (2.14) we see that the second term on the right-hand side of the last inequality is bounded by a constant times the $L^{1}$ norm of $f$. Moreover, since $S_{n}=\left\{h_{n} \neq 0\right\} \subset B_{\rho}\left(x_{n}, C r_{n}\right) \subset B_{d}\left(x_{n}, C C_{2} r_{n}\right)$, we can apply (4.3) in order to obtain

$$
\begin{aligned}
\mu(\{x & \left.\left.\notin \bigcup_{n} B_{d}\left(x_{n}, M C C_{2} r_{n}\right):\left|T\left(\sum h_{n}\right)(x)\right|>C_{0} D\right\}\right) \\
& \leqslant c \sum_{i} \int_{X-B_{d}\left(x_{i}, M C C_{2} r_{i}\right)}\left|T h_{i}(x)\right| d \mu(x) \\
& \leqslant c \sum_{i} \int_{X}\left|h_{i}(x)\right| d \mu(x) \leqslant C\|f\|_{1} .
\end{aligned}
$$

The $L^{p}$ boundedness of $T$ is a consequence of the Marcinkiewicz interpolation theorem.

(4.4) Corollary. Let $(X, d, \mu)$ be a space of homogeneous type such that continuous functions are dense in $L^{1}$. Let $\left\{K_{\alpha}: \alpha \in \Gamma\right\}$ be a family of measurable functions defined on $X \times X$ such that

$$
T f(x)=\sup _{\alpha \in \Gamma}\left|\int_{X} K_{\alpha}(x, y) f(y) d \mu(y)\right|
$$

defines a measurable function on $X$, whenever $f \in L^{1}+L^{\infty}$. Assume 
(4.5) there exists $C$ independent of $x$ and $\alpha$ such that

$$
\int_{X}\left|K_{\alpha}(x, y)\right| d \mu(y) \leqslant C,
$$

(4.6) there exist two constants $M$ and $C$ such that

$$
\int_{d(z, y) \geqslant M d(x, y)} \sup _{\alpha \in \Gamma}\left|K_{\alpha}(z, y)-K_{\alpha}(z, x)\right| d \mu(z) \leqslant C,
$$

for every $x, y \in X$.

Then $T$ is of weak type $(1,1)$ and of strong type $(p, p)(1<p \leqslant \infty)$.

Proof. We only need to check (4.3). Let $h \in L^{1}$ such that $\{h \neq 0\} \subset B\left(x_{0}, r\right)$ and $\int h d \mu=0$. We have

$$
\begin{aligned}
\int_{X-B\left(x_{0}, M r\right)}|T h(z)| d \mu(z) \\
=\int_{X-B\left(x_{0}, M r\right)} \sup _{\alpha \in \Gamma}\left|\int_{B\left(x_{0}, r\right)} h(y)\left[K_{\alpha}(z, y)-K_{\alpha}\left(z, x_{0}\right)\right] d \mu(y)\right| d \mu(z) \\
\leqslant \int_{B\left(x_{0}, r\right)}|h(y)| \int_{X-B\left(x_{0}, M r\right)} \sup _{\alpha \in \Gamma}\left|K_{\alpha}(z, y)-K_{\alpha}\left(z, x_{0}\right)\right| d \mu(z) d \mu(y) \\
\leqslant C\|h\|_{1} .
\end{aligned}
$$

Now, we apply this result to the study of a class of approximate identities on normal spaces of homogeneous type. From the $\mathbf{R}^{n}$ version of Corollary (4.4) it follows that $|\nabla k(x)| \leqslant C|x|^{-n-1}$ is a sufficient condition in order that the family $k_{\varepsilon}(x, y)=\varepsilon^{-n} k\left(\varepsilon^{-1}(x-y)\right)$ defines a well-behaved approximate identity. The class of approximate identities which we shall consider arises quite naturally by the following consideration.

Propositron. The following conditions on a function $l(x)$ defined on $\mathbf{R}^{n}$ are equivalent:

(a) $l(x) \in \mathscr{C}^{1}\left(\mathbf{R}^{n}-\{0\}\right)$ is a nonnegative, integrable and radial function such that

$$
|\nabla l(x)| \leqslant C \cdot|x|^{-n-1}
$$

for some finite constant $C$ and every $x \in \mathbf{R}^{n}-\{0\}$.

(b) There exists $\phi \geqslant 0$ defined on $\mathbf{R}^{+}$such that

$$
\begin{gathered}
l(x)=\phi\left(|x|^{n}\right)|x|^{-n}, \\
\phi \in \mathscr{C}^{1}\left(\mathbf{R}^{+}\right) \cap L^{\infty}\left(\mathbf{R}^{+}\right), \\
\left|\phi^{\prime}(t)\right| \leqslant C t^{-1}, \\
\int_{0}^{\infty} \phi(t) t^{-1} d t<\infty .
\end{gathered}
$$

In the approach (b) $l(x-y)$ is regarded as a function of $|x-y|^{n}$, the normalized quasi-distance on the $n$-dimensional euclidean space. Moreover, (4.9)-(4.11) are properties of $\phi$ as a real function, and so independent of the euclidean structure of $\mathbf{R}^{n}$. The associated family of kernels is

$$
l_{\delta}(x-y)=\delta^{-n} l((x-y) / \delta)=\phi\left(\delta^{-n}|x-y|^{n}\right)|x-y|^{-n},
$$

where $\delta>0$. 
Consequently, a general class of approximate identities on normal spaces of homogeneous type is obtained considering families of kernels given by

$$
K_{\varepsilon}(x, y)=\phi\left(\varepsilon^{-1} d(x, y)\right) d(x, y)^{-1}
$$

for $\varepsilon>0$; where $\phi$ is a function satisfying (4.9)-(4.11).

In the sequel, $(X, d, \mu)$ denotes a normal space of homogeneous type such that continuous functions are dense in $L^{1}$. The next lemma shows that (4.5) holds for the family $\left\{K_{\epsilon}\right\}$ defined by (4.12), provided the space is endowed with property $\mathrm{P}$.

(4.13) Lemma. Let $\phi$ be a nonnegative function defined on $\mathbf{R}^{+}$satisfying (4.9)-(4.11), and let $K_{\varepsilon}$ be defined as in (4.12). If $(X, d, \mu)$ satisfies property $\mathrm{P}$, then there exists a constant $C$ such that

$$
\int_{d(x, y)<a} K_{\varepsilon}(x, y) d \mu(y) \leqslant C \int_{0}^{a / \varepsilon} \phi(t) t^{-1} d t
$$

holds for every $\varepsilon \in(0,1), x \in X$ and $a>0$. Consequently,

$$
\int_{X} K_{\varepsilon}(x, y) d \mu(y) \leqslant C \int_{0}^{\infty} \phi(t) t^{-1} d t .
$$

Proof. If $x$ is a fixed point in $X$, then $K_{\varepsilon}(x, y)$ is measurable as a function of $(\varepsilon, y)$ defined in $(0,1) \times X$. Since $(X, \mu)$ is a $\sigma$-finite measure space, we have

$$
\int_{0}^{1}\left\{\int_{X} K_{\varepsilon}(x, y) d \mu(y)\right\} d \varepsilon=\int_{X} d(x, y)^{-1}\left\{\int_{0}^{1} \phi\left(\varepsilon^{-1} d(x, y)\right) d \varepsilon\right\} d \mu(y) .
$$

If we set $t=\varepsilon^{-1} d(x, y)$, changing again the order of integration, we get

$$
\begin{aligned}
\int_{0}^{1}\left\{\int_{X} K_{\varepsilon}(x, y) d \mu(y)\right\} d \varepsilon & =\int_{0}^{\infty} \phi(t) t^{-2} \mu(B(x, t)) d t \\
& \leqslant C \int_{0}^{\infty} \phi(t) t^{-1} d t<\infty .
\end{aligned}
$$

Therefore $\int_{B(x, a)} K_{\varepsilon}(x, y) d \mu(y)$ is integrable as a function of $\varepsilon \in(0,1)$. Then we can apply the Lebesgue differentiation theorem in order to obtain

$$
\int_{B(x, a)} K_{\delta}(x, y) d \mu(y)=\lim _{h \rightarrow 0} h^{-1} \int_{\delta}^{\delta+h}\left\{\int_{B(x, a)} K_{\varepsilon}(x, y) d \mu(y)\right\} d \varepsilon .
$$

In order to estimate the right-hand side we write

$$
\begin{aligned}
& h^{-1} \int_{\delta}^{\delta+h}\left\{\int_{d(x, y)<a} K_{\varepsilon}(x, y) d \mu(y)\right\} d \varepsilon \\
&=h^{-1} \int_{d(x, y)<a} d(x, y)^{-1}\left\{\int_{\delta}^{\delta+h} \phi\left(\varepsilon^{-1} d(x, y)\right) d \varepsilon\right\} d \mu(y) \\
&=h^{-1} \int_{d(x, y)<a}\left\{\int_{d(x, y) /(\delta+h)}^{d(x, y) / \delta} \phi(t) t^{-2} d t\right\} d \mu(y) \\
&=h^{-1} \int_{a /(\delta+h)}^{a / \delta} \phi(t) t^{-2}[\mu(B(x, a))-\mu(B(x, t \delta))] d t \\
&+h^{-1} \int_{0}^{a /(\delta+h)} \phi(t) t^{-2}[\mu(B(x, t(\delta+h)))-\mu(B(x, t \delta))] d t .
\end{aligned}
$$


Taking into account property $\mathrm{P}$, we get

$$
h^{-1} \int_{\delta}^{\delta+h}\left\{\int_{d(x, y)<a} K_{\varepsilon}(x, y) d \mu(y)\right\} d \varepsilon \leqslant C \int_{0}^{a / \delta} \phi(t) t^{-1} d t .
$$

Thus, (4.14) is proved for almost every $\varepsilon \in(0,1)$. Let $\delta \in(0,1)$. Taking $\varepsilon>\delta$ such that (4.14) holds for $\varepsilon$, from (4.9) and (4.10) it follows that for every $N \in \mathbf{N}$,

$$
\begin{aligned}
\int_{N^{-1} \leqslant d(x, y)<a} & K_{\delta}(x, y) d \mu(y) \\
\leqslant & \int_{N^{-1} \leqslant d(x, y)<a} d(x, y)^{-1}\left|\phi\left(\delta^{-1} d(x, y)\right)-\phi\left(\varepsilon^{-1} d(x, y)\right)\right| d \mu(y) \\
& +\int_{N^{-1} \leqslant d(x, y)<a} K_{\varepsilon}(x, y) d \mu(y) \\
\leqslant & C a N \varepsilon \frac{\varepsilon-\delta}{\varepsilon \delta}+C \int_{0}^{a / \delta} \phi(t) t^{-1} d t
\end{aligned}
$$

holds. Since we can choose $\varepsilon-\delta$ arbitrarily small, clearly

$$
\int_{N^{-1} \leqslant d(x, y)<a} K_{\delta}(x, y) d \mu(y) \leqslant C \int_{0}^{a / \delta} \phi(t) t^{-1} d t
$$

uniformly on $N$. Therefore (4.14) holds for every $\varepsilon \in(0,1)$, and the lemma is proved.

REMARK. The result of this lemma remains valid if instead of (4.10) we only know that the function $\phi$ is of class Lipschitz 1 on each interval of the form $(t, \infty)$, with Lipschitz norm bounded by $C t^{-1}$.

(4.16) THEOREM. Let $(X, d, \mu)$ be a space of order $\alpha$ satisfying property $\mathrm{P}$ and $K_{\varepsilon}$ defined by (4.12). Then the operator

$$
T f(x)=\sup _{0<\varepsilon<1}\left|\int_{X} K_{\varepsilon}(x, y) f(y) d \mu(y)\right|
$$

is of weak type $(1,1)$ and of strong type $(p, p)(1<p \leqslant \infty)$.

Proof. In order to apply Corollary (4.4) we observe that by the preceding lemma the property (4.5) on uniform integrability of $\left\{K_{\varepsilon}\right\}$ holds. We now prove that (4.6) is also valid. First observe that $\sup _{0<\varepsilon<1}\left|K_{\varepsilon}(z, y)-K_{\varepsilon}(z, x)\right|$ is measurable as a function of $z$ for fixed $x, y \in X$. On account of the property (4.9) of $\phi$ we get the following estimate:

$$
\begin{aligned}
\left|K_{\varepsilon}(z, y)-K_{\varepsilon}(z, x)\right| \leqslant & d(z, y)^{-1}\left|\phi\left(\varepsilon^{-1} d(z, y)\right)-\phi\left(\varepsilon^{-1} d(z, x)\right)\right| \\
& +\left|\phi\left(\varepsilon^{-1} d(z, x)\right)\right|\left|d(z, y)^{-1}-d(z, x)^{-1}\right| \\
\leqslant & \left|\phi^{\prime}(\xi)\right||d(z, y)-d(z, x)| \varepsilon^{-1} d(z, y)^{-1} \\
& +C|d(z, y)-d(z, x)| d(z, y)^{-1} d(z, x)^{-1}
\end{aligned}
$$


where $\xi$ is a real number between $d(z, y) \varepsilon^{-1}$ and $d(z, x) \varepsilon^{-1}$. If $z$ is such that $d(z, x) \geqslant 2 k d(x, y)$, then $d(z, x) \leqslant 2 k d(z, y)$ and, consequently,

$$
\xi \geqslant d(x, z) \varepsilon^{-1}(2 k)^{-1} \text {. }
$$

Therefore from (4.10) we deduce that

$$
\begin{aligned}
\left|K_{\varepsilon}(z, y)-K_{\varepsilon}(z, x)\right| & \leqslant C|d(z, y)-d(z, x)| d(z, y)^{-1} d(z, x)^{-1} \\
& \leqslant C|d(z, y)-d(z, x)| d(z, x)^{-2} .
\end{aligned}
$$

Moreover, since $d(z, x) \geqslant 2 k d(x, y)$ also implies $d(z, y) \leqslant C d(z, x)$, using the fact that the distance $d$ is of order $\alpha$, we get

$$
\left|K_{\varepsilon}(z, y)-K_{\varepsilon}(z, x)\right| \leqslant C d(x, y)^{\alpha} d(z, x)^{-1-\alpha}
$$

for every $\varepsilon>0$. Then (4.6) follows from (2.19). It remains only to prove that $T f(x)$ is a measurable function. Even more, we shall see that if $f$ is bounded and integrable, then $T f(x)$ is lower semicontinuous. Let $\lambda>0, U_{\lambda}=\{x \in X: T f(x)>$ $\lambda\}$ and $x_{0} \in U_{\lambda}$. Then there exists $\varepsilon \in(0,1)$ such that $\left|\int K_{\varepsilon}\left(x_{0}, z\right) f(z) d \mu(z)\right|>\lambda$. Let $x \in X$ such that $2 k d\left(x, x_{0}\right)<1$. Applying (4.14) and (4.17) we see that

$$
\begin{aligned}
\mid \int_{X}\left[K_{\varepsilon}\left(x_{0}, z\right)-\right. & \left.K_{\varepsilon}(x, z)\right] f(z) d \mu(z) \mid \\
\leqslant & \int_{d\left(z, x_{0}\right) \geqslant\left[2 k d\left(x, x_{0}\right)\right]^{1 / 2}}\left|K_{\varepsilon}\left(x_{0}, z\right)-K_{\varepsilon}(x, z)\right||f(z)| d \mu(z) \\
& +\int_{d\left(z, x_{0}\right)<\left[2 k d\left(x, x_{0}\right)\right]^{1 / 2}}\left[K_{\varepsilon}\left(x_{0}, z\right)+K_{\varepsilon}(x, z)\right]|f(z)| d \mu(z) \\
\leqslant & C d\left(x, x_{0}\right)^{\alpha}\|f\|_{\infty} \int_{d\left(z, x_{0}\right) \geqslant\left[2 k d\left(x, x_{0}\right)\right]^{1 / 2}} d\left(z, x_{0}\right)^{-1-\alpha} d \mu(z) \\
& +C\|f\|_{\infty} \int_{0<t<k\left[\left(2 k d\left(x, x_{0}\right)\right)^{1 / 2}+d\left(x, x_{0}\right)\right] \varepsilon^{-1}} \phi(t) t^{-1} d t \\
\leqslant & C\|f\|_{\infty} d\left(x, x_{0}\right)^{\alpha / 2}+C\|f\|_{\infty} \int_{0<t<C \varepsilon^{-1} d\left(x, x_{0}\right)^{1 / 2}} \phi(t) t^{-1} d t .
\end{aligned}
$$

Then $\int\left[K_{\varepsilon}\left(x_{0}, z\right)-K_{\varepsilon}(x, z)\right] f(z) d \mu(z)$ tends to 0 when $d\left(x, x_{0}\right)$ tends to 0 , consequently the inequality $\left|\int K_{\varepsilon}(x, z) f(z) d \mu(z)\right|>\lambda$ holds for every $x$ in a neighborhood of $x_{0}$.

REMARK 1. This result remains valid if $\phi$ satisfies the weaker smoothness hypothesis introduced in the remark after Lemma (4.13). Then if $\Psi: \mathbf{R}^{+} \rightarrow \mathbf{R}^{+} \cup\{0\}$ is a nonincreasing integrable function, we can apply Theorem (4.16) with $\phi(t)=$ $\int_{t / 2}^{t} \Psi(s) d s$. In this way, from our result, we can deduce an extension to spaces of homogeneous type of a classical result due to Calderón and Zygmund. More precisely, if $\Psi: \mathbf{R}^{+} \rightarrow \mathbf{R}^{+} \cup\{0\}$ is a nonincreasing integrable function, then the maximal operator associated with the family of kernels

$$
K_{\varepsilon}(x, y)=\varepsilon^{-1} \Psi\left(\varepsilon^{-1} d(x, y)\right)
$$

is of weak type $(1,1)$ and of strong type $(p, p), 1<p \leqslant \infty$. 
REMARK 2 . The symmetry of the quasi-distance $d$ can be replaced by

$$
C^{-1} d(x, y) \leqslant d(y, x) \leqslant C d(x, y),
$$

where $C$ is a finite constant. Then Example 5 in $\$ 1$ falls into the scope of Theorem (4.16).

Next, we give examples of normal spaces of homogeneous type of order $\alpha$ and functions $\phi$ satisfying (4.9)-(4.11) but such that the boundedness property (4.5) is not valid. This shows that some additional property, replacing $\mathrm{P}$, is necessary in order to obtain the $L^{\infty}$ boundedness of the maximal operator.

EXAMPLE 1. Let $\rho: \mathbf{R} \rightarrow \mathbf{R}^{+} \cup\{0\}$ be the function defined by

$$
\rho(x)= \begin{cases}0 & \text { if } x=0, \\ 2|x|-2^{j} & \text { if } 2^{j} \leqslant|x|<3 \cdot 2^{j-1}, \\ 2^{j+1} & \text { if } 3 \cdot 2^{j-1} \leqslant|x|<2^{j+1} .\end{cases}
$$

If $X=\mathbf{R}, \mu$ is the Lebesgue measure and $d(x, y)=\rho(x-y)$, then $(X, d, \mu)$ is a normal space of order 1. Let $\phi: \mathbf{R}^{+} \rightarrow \mathbf{R}^{+} \cup\{0\}$ be the function defined by

$$
\phi(t)= \begin{cases}0 & \text { if } t \leqslant 12, \\ 2^{-i} t+i^{-1}-1 & \text { if } t \in\left(2^{i}(1-1 / i), 2^{i}\right), i \geqslant 4, \\ -2^{-i} t+i^{-1}+1 & \text { if } t \in\left(2^{i}, 2^{i}(1+1 / i)\right), \\ 0 & \text { if } t \in\left(2^{i}(1+1 / i), 2^{i+1}(1-1 /(i+1))\right) .\end{cases}
$$

Clearly $\phi$ is bounded and $\left|\phi^{\prime}(t)\right| \leqslant C t^{-1}$. Moreover

$$
\begin{aligned}
\int_{0}^{\infty} t^{-1} \phi(t) d t & =\sum_{i=4}^{\infty}\left\{\int_{2^{i}(1-1 / i)}^{2^{i}}\left[2^{-i}+\left(i^{-1}-1\right) t^{-1}\right] d t\right. \\
& \left.+\int_{2^{i}}^{2^{i}(1+1 / i)}\left[-2^{-i}+\left(i^{-1}+1\right) t^{-1}\right] d t\right\} \\
& =\sum_{i=4}^{\infty}\left\{i^{-1} \log [(1+1 / i) /(1-1 / i)]+\log \left[1-(1 / i)^{2}\right]\right\} \\
& \leqslant C \sum_{i=4}^{\infty} i^{-2}<\infty .
\end{aligned}
$$

Let $\varepsilon_{j}=2^{-j}$ and $K_{\varepsilon_{j}}(0, y)=d(0, y)^{-1} \phi\left[\varepsilon_{j}^{-1} d(0, y)\right]$. Then

$$
\begin{aligned}
\int_{X} K_{\varepsilon_{j}}(0, y) d \mu(y) & \geqslant \sum_{i=-\infty}^{\infty} \int_{\left\{y: d(0, y)=2^{i}\right\}} 2^{-i} \phi\left[2^{j+i}\right] d y \\
& =C \sum_{i \geqslant 4-j}(i+j)^{-1}=\infty .
\end{aligned}
$$

Therefore $(\mathbf{R}, d, \mu)$ is a normal space of order $\alpha$, and the kernel $K_{\varepsilon}$ satsifies (4.9)-(4.11); however, (4.5) fails.

In the preceding example, the measure and the quasi-distance are both translation invariant, therefore, the set of those $\varepsilon>0$ for which $\int K_{\varepsilon}(x, y) d y$ is finite, is independent of $x$. We could set the question whether it is possible to choose a particular sequence for which (4.5) holds. To answer this we give an example where 
the sets

$$
E_{x}=\left\{\varepsilon \in(0,1): \int_{\mathbf{R}} K_{\varepsilon}(x, y) d y<\infty\right\}
$$

are such that $\bigcap_{x} E_{x}=\varnothing$.

ExAmple 2. Let $X=\mathbf{R}, \mu$ be the Lebesgue measure and $\rho$ be as in Example 1. Denote

$$
\begin{aligned}
R_{1}= & \left\{(x, y) \in \mathbf{R}^{2}:-\frac{1}{2}<x<\frac{1}{2}, x \leqslant y \leqslant 2\right\}, \\
R_{2}= & \left\{(x, y) \in \mathbf{R}^{2}: \frac{1}{2} \leqslant x \leqslant 1, x \leqslant y \leqslant 2\right\}, \\
R_{3}= & \left\{(x, y) \in \mathbf{R}^{2}:-1 \leqslant x \leqslant-\frac{1}{2}, x \leqslant y \leqslant 2\right\} \\
& \cup\left\{(x, y) \in \mathbf{R}^{2}:-1 \leqslant x \leqslant 1,2 \leqslant y \leqslant \frac{5}{2}\right\}, \\
R_{4}= & \left\{(x, y) \in \mathbf{R}^{2}: x<y\right\}-\bigcup_{i=1}^{3} R_{i} .
\end{aligned}
$$

Let $\Psi: \mathbf{R}^{2} \rightarrow[1 / 2,1]$ be a symmetric Lipschitz function such that

$$
\Psi(x, y)= \begin{cases}\frac{1}{2} & \text { if }(x, y) \in R_{1} \\ 1 & \text { if }(x, y) \in R_{4} \\ x & \text { if }(x, y) \in R_{2}\end{cases}
$$

If we set $d(x, y)=\rho(x-y) \Psi(x, y)$, then $(X, d, \mu)$ is a normal space of homogeneous type of order 1 . Let $\phi$ be defined by

$$
\phi(t)= \begin{cases}0 & \text { if } t \geqslant 1 / 6, \\ 2^{i} t+i^{-1}-1 & \text { if } t \in\left(2^{-i}(1-1 / i), 2^{-i}\right), i \geqslant 3, \\ -2^{i} t+i^{-1}+1 & \text { if } t \in\left(2^{-i}, 2^{-i}(1+1 / i)\right), \\ 0 & \text { if } t \in\left(2^{-(i+1)}(1+1 /(i+1)), 2^{-i}(1-1 / i)\right) .\end{cases}
$$

As in Example 1 it is easy to check that the function $\phi$ satisfies (4.9)-(4.11). However if $\varepsilon \in(0,1)$, then there exist $x \in[1 / 2,1)$ and $j \in \mathbf{N} \cup\{0\}$ such that $\varepsilon=x 2^{-j}$. Therefore

$$
\begin{aligned}
\int_{\mathbf{R}} K_{\varepsilon}(x, y) d y & \geqslant \sum_{i=-\infty}^{+\infty} \int_{\left\{y: d(x, y)=\varepsilon 2^{-i}\right\}} K_{\varepsilon}(x, y) d y \\
& \geqslant \sum_{i \geqslant 3} i^{-1} 2^{i} \varepsilon^{-1}\left|\left\{y: \rho(x-y) \Psi(x, y)=x 2^{-i-j}\right\}\right| \\
& \geqslant \sum_{i \geqslant 3} i^{-1} 2^{i+j} \mid\left\{y: 2>y>x \text { and } \rho(y-x)=2^{-i-j}\right\} \mid \\
& \geqslant C \sum_{i \geqslant 3} i^{-1}=\infty .
\end{aligned}
$$

This implies that $\bigcap_{x} E_{x}=\varnothing$. 


\section{REFERENCES}

[CG] R. Coifman and M. de Guzmán, Singular integrals and multipliers on homogeneous spaces, Rev. Un. Mat. Argentina 25 (1970), 137-144.

[CW] R. Coifman and G. Weiss, Analyse harmonique non-commutative sur certains espaces homogènes, étude de certaines intégrales singulières, Lecture Notes in Math., vol. 242, Springer-Verlag, Berlin, 1971.

[G] M. de Guzman, Real variable methods in Fourier analysis, Notas de Matemática (75), North-Holland Math. Studies, No. 46, North-Holland, Amsterdam. 1981.

[GGW] A. Gatto, C. Gutierrez and R. Wheeden, On weighted fractional integrals, Conference on Harmonic Analysis, In Honor of Antoni Zygmund, Wadsworth, Belmont, Calif., 1983, pp. 124-137.

[MS1] R. Macias and C. Segovia, Lipschitz functions on spaces of homogeneous type, Adv. in Math. 33 (1979), 257-270.

[MS2] _ A decomposition into atoms of distributions on spaces of homogeneous type, Adv. in Math. 33 (1979), 271-309.

[R] N. Riviere, Singular integrals and multiplier operators, Ark. Mat. 9 (1973), 243-270.

[Z] F. Zó, A note on the approximation of the identity, Studia Math. 55 (1976), 111-122.

Programa Especial de Matematica Aplicada, Conicet C.C. No. 91, 3000 Santa Fe, Argentina.

Current address: School of Mathematics, University of Minnesota, 127 Vincent Hall, 206 Church Street S. E., Minneapolis, Minnesota 55455 